\title{
Zur Messung der Steuerbelastung von Unternehmen
}

\author{
Ansätze unter besonderer Berücksichtigung der österreichischen \\ Volkswirtschaftlichen Gesamtrechnungen
}

\author{
Thomas Kostal \\ Institut für Finanzwissenschaft \\ Wirtschaftsuniversität Wien
}

\begin{abstract}
Zusammenfassung: Zur Messung der (effektiven) Steuerbelastung von Unternehmen stehen mikroökonomische Methoden makroökonomischen Ansätzen auf Basis der VGR (implizite Steuerquoten) gegenüber. Beide Zugänge liefern Antworten auf unterschiedliche Fragestellungen, ihre Ergebnisse sind daher nicht vergleichbar. Während für die Berechnung mikroökonomischer (Grenz-) Steuerquoten eine akzeptierte Methode vorliegt, ist die Ermittlung impliziter Steuerquoten von Kapitaleinkommen problematisch und ihre Interpretation schwierig. Als Indikator für die Steuerbelastung von Unternehmenseinkommen ist die ,einfache“ implizite Steuerquote kaum geeignet. Auf Basis der österreichischen VGR werden Bereinigungsmaßnahmen diskutiert, die eine Möglichkeit bieten, die tatsächliche (durchschnittliche) Steuerbelastung der Einkünfte aus Unternehmertätigkeit zu ermitteln. Angesichts der derzeitigen Datenlage der offiziellen Statistik in Österreich erscheinen diese Bereinigungen jedoch nur mit Einschränkungen möglich.
\end{abstract}

\begin{abstract}
Basically, there are two different kinds of approaches to calculate the effective tax burden on companies. One is based on microeconomic methods, the other bears on the national accounts (implicit tax rates). Both of them provide answers to different questions, thus making the results incomparable. While the calculation of (microeconomic) marginal effective tax rates is widely accepted, the calculation of implicit tax rates on capital income is problematic and its interpretation difficult. The "simple" implicit tax rate is hardly apt to indicate the tax burden on company incomes. Some clarification measures are being discussed on the basis of the Austrian national accounts, thus making it possible to calculate the effective (average) tax burden on company incomes. However, with regard to the official $\mathrm{Au}-$ strian statistics currently available such measures seem to be possible with restrictions only.
\end{abstract}

Schlüsselwörter: Steuerbelastung von Unternehmen, Effektive Steuersätze, Implizite Steuerquoten, Volkswirtschaftliche Gesamtrechnungen.

\section{Einleitung}

Die Frage nach der Steuerbelastung von Unternehmen (-seinkünften) weist nicht nur in Zeiten von Steuerreformen und zunehmendem internationalen Steuerwettbewerb wirtschaftspolitische Brisanz auf. Genährt wird diese Brisanz vor allem durch stark divergierende Ergebnisse der wenigen bisher vorliegenden empirischen Studien. Erinnert sei 
etwa an die intensive Diskussion über die tatsächliche Höhe der Unternehmenssteuerlast in Deutschland Ende der 80er Jahre (Fuest and Kroker, 1989). Zum Großteil resultieren die ermittelten - beachtlichen - Unterschiede in der Höhe der Steuerlast vor allem aus der gewählten Berechnungsmethode: mikroökonomisch orientierte Methoden stehen makroökonomischen Ansätzen gegenüber. Es zeigt sich, daß die Wahl einer bestimmten Methode das Ergebnis entscheidend beeinflußt und daher nur Antworten auf bestimmte Fragen zuläßt. Eine allgemein gültige Methode der Messung der effektiven Steuerbelastung von Unternehmen gibt es nicht.

Mit den volkswirtschaftlichen Gesamtrechnungen (VGR) steht den Wirtschaftswissenschaften ein bewährtes Informationssystem zur Verfügung. Die Daten der VGR werden auch regelmäßig zur Ermittlung von Steuerquoten herangezogen. Die globale Steuerquote zeigt beispielsweise die (durchschnittliche) Gesamtbelastung einer Volkswirtschaft durch das jeweilige Steuersystem. Dabei handelt es sich natürlich nicht um die endgültige Belastung (Entzug von Ressourcen), sondern lediglich um jenen Anteil am BIP, der nicht unmittelbar zur Disposition durch die Wirtschaftssubjekte zur Verfügung steht.

Darüber hinaus lassen sich auch spezifische Steuerquoten berechnen. Diese stellen die Verteilung der gesamtwirtschaftlichen Steuerbelastung auf einzelne volkswirtschaftliche Aggregate, wie beispielsweise Arbeit, Kapital und Konsum, dar.

Mangels einer eigenen Sektorengliederung der (österreichischen) VGR in Unternehmen, Haushalte, Staat und Ausland ist eine direkte Ermittlung der Steuerbelastung makroökonomischer Sektoren nicht möglich. Das neue Europäische System Volkswirtschaftlicher Gesamtrechnungen (ESVG 1995) sieht zwar eine Gliederung in die Sektoren nichtfinanzielle Kapitalgesellschaften, finanzielle Kapitalgesellschaften, Staat, private Haushalte, private Organisationen ohne Erwerbszweck und übrige Welt vor. Die methodische Einschränkung einer nicht möglichen Trennung der ,echten“ Besitzeinkünfte der Haushalte von den im Haushaltssektor miterfaßten Einkünften bestimmter Unternehmenstätigkeiten (insbesondere selbständig Gewerbetreibender) bleibt jedoch bestehen.

Dennoch erscheint es lohnenswert der Frage nachzugehen, ob die VGR nicht durch „Bereinigungen“ eine geeignete Grundlage für die Ermittlung der Steuerbelastung der Unternehmenseinkünfte bieten können. Gelänge es beispielsweise, die in der Verteilungsrechnung ausgewiesenen „Einkünfte aus Besitz und Unternehmung“ um alle Besitzeinkünfte, die nicht dem Unternehmenssektor zufließen, zu bereinigen, hätte man eine solche Basis. Die Interpretation der so ermittelbaren Steuerquote ist aber nicht unproblematisch. Insbesondere ist dabei auf die Konventionen der (österreichischen) VGR abzustellen. Die Analyse dieser Frage, aber auch die Behandlung der damit verbundenen Probleme stehen im Mittelpunkt der vorliegenden Untersuchung.

Im Einzelnen gliedert sich die Arbeit folgendermaßen. Nach einem Überblick über die unterschiedlichen Möglichkeiten der Messung der Steuerbelastung von Unternehmen (Abschnitt 2) widmet sich Abschnitt 3 im Detail jenen Steuerquoten, die sich aus den VGR ableiten lassen. Dabei wird insbesondere auf die Ermittlungsmethoden und auf die Abgrenzung der Unternehmenseinkünfte in den österreichischen VGR eingegangen und damit zusammenhängende Interpretationsschwierigkeiten aufgezeigt. Anschließend werden Bereinigungsmaßnahmen vorgeschlagen, die notwendig wären, um zu einer praktikablen Abgrenzung der tatsächlichen Unternehmenseinkommen und der korrespondierenden Steuern zu gelangen. Insbesondere werden auch die (statistischen) Grenzen solcher 
Bereinigungsmaßnahmen und die Interpretationsschwierigkeiten der so ermittelten Steuerquote aufgezeigt. Ein Resümee beschließt die Arbeit (Abschnitt 4).

\section{Methodenüberblick}

In der einschlägigen Literatur finden sich verschiedene Ansätze zur Messung der Steuerbelastung von Unternehmen (Callihan, 1994). Folgende grundlegenden Unterscheidungen bezüglich der Methoden der Steuerbelastungsmessung können getroffen werden:

- nominale Tarifbelastung versus effektive Steuerbelastung

- Steuerbelastung einzelner Unternehmen versus Steuerbelastung des gesamten Unternehmenssektors einer Volkswirtschaft

- Ex post-Steuerbelastung auf Basis historisch-empirischer Daten versus Ex anteSteuerbelastung auf Basis von Modellrechnungen

- marginale Steuerbelastung versus durchschnittliche Steuerbelastung

Ein bestimmter Ansatz läßt sich durch mehrere der angeführten Unterscheidungsmerkmale charakterisieren. Beispielsweise handelt es sich bei Modellrechnungen regelmäßig um mikroökonomische Ansätze, die die effektive marginale Steuerbelastung repräsentativer Unternehmen berechnen. Die VGR hingegen zielen auf eine Berechnung der durchschnittlichen effektiven Steuerbelastung des gesamten Unternehmenssektors ab. Daneben lassen sich noch die (nominale) Tarifbelastung von Unternehmensgewinnen und die Berechnung der durchschnittlichen Steuerbelastung ausgewählter Unternehmen auf Basis ihrer Bilanzdaten unterscheiden (Abbildung 1).

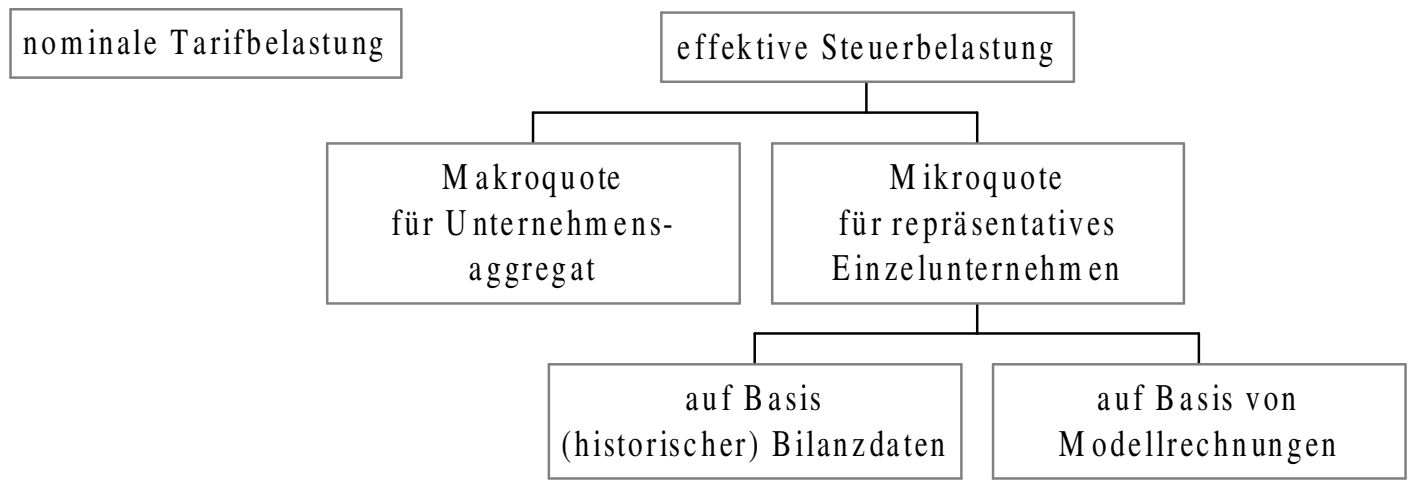

Abbildung 1: Ansätze zur Ermittlung der Steuerbelastung von Unternehmen

\subsection{Nominale Tarifbelastung}

Die Verwendung der nominalen Tarifbelastung von Unternehmensgewinnen, beispielsweise des Körperschaftsteuersatzes für Kapitalgesellschaften, stellt die einfachste Art zur Ermittlung der Unternehmenssteuerbelastung dar. Die Aussagekraft dieser Kennzahl ist 
jedoch stark eingeschränkt, da sie lediglich auf die gesetzlich normierten Steuersätze abstellt, ohne die Ermittlung der jeweiligen Bemessungsgrundlage miteinzubeziehen. Abschreibungsregelungen, steuerliche Investitionsbegünstigungen etc., die die Bemessungsgrundlage schmälern, werden nicht berücksichtigt. Dementsprechend ist damit die effektive Höhe der Steuerbelastung eines Unternehmens nicht abbildbar.

Darüber hinaus sind auch Aussagen über die gesamte Steuerbelastung von Unternehmenseinkünften nicht möglich, da diese regelmäßig durch mehrere Steuerarten mit unterschiedlichen Tarifen belastet werden (Körperschaftsteuer, Kapitalertragsteuer etc.).

Die Verwendung der nominalen Tarifbelastung erscheint daher - vor allem aufgrund ihrer leichten Verfügbarkeit - bei internationalen Steuerbelastungsvergleichen lediglich als erste Orientierung hilfreich. Sie kann auch Anhaltspunkte über die steuerpolitische „Absicht“ des Gesetzgebers und deren Änderung im Zeitablauf liefern. Dabei besteht jedoch die Gefahr, daß bei Vorlage umfangreicher Ausnahmeregelungen für die Ermittlung der Bemessungsgrundlage eine „negative“ Steuerillusion hervorgerufen wird (Littmann, 1987, S. 9).

Im Gegensatz zur nominalen Tarifbelastung versuchen alle im Folgenden behandelten Steuerquoten die tatsächliche (effektive) Steuerbelastung darzustellen. Allen effektiven Steuerbelastungsquoten ist es gemeinsam, daß sie die Steuern nicht auf die jeweilige steuerrechtliche Bemessungsgrundlage beziehen, sondern auf eine ökonomisch relevante Größe, die die wirtschaftliche Leistungsfähigkeit des einzelnen Wirtschaftssubjektes (Mikroquoten) beziehungsweise des Wirtschaftsaggregates (Makroquoten) widerspiegeln soll. Es soll damit das tatsächliche Ausmaß der materiellen Einkommenskürzung durch die Besteuerung ausgedrückt werden.

\subsection{Mikroökonomische Steuerquoten}

Das Charakteristikum der mikroökonomischen Steuerquoten ist, daß sie auf die Betrachtung einzelner Unternehmen abstellen. Grundsätzlich ist dabei zwischen der Steuerbelastung repräsentativ ausgewählter Unternehmen auf Basis historischer Bilanzdaten und der modelltheoretischen Analyse eines idealtypischen Unternehmens (eines zusätzlichen Investitionsprojektes) unter Anwendung des geltenden Steuerrechts zu unterscheiden.

\subsubsection{Die durchschnittliche ex post-Steuerbelastung}

Eine Möglichkeit die effektive Steuerbelastung zu ermitteln, ist die Berechnung der durchschnittlichen jährlichen Steuerbelastung eines oder mehrerer Unternehmen(s). Von der Grundüberlegung her ist diese Quote einfach zu ermitteln. Sie errechnet sich als Quotient aus jährlicher Steuerleistung und Gewinn des Unternehmens im betreffenden Jahr.

Abgesehen von der methodisch und empirisch meist schwierigen Auswahl repräsentativer Unternehmen liegt die Problematik dieser Methode in ihrer konkreten Umsetzung, sowohl hinsichtlich der Abgrenzung der einzubeziehenden Unternehmenssteuern - Sollen neben den Unternehmensertragsteuern auch die Unternehmenssubstanzsteuern und/oder die Steuern auf der Ebene der Kapitalgeber miteinbezogen werden? (Schaden, 1995, S. 53ff) - als auch in der Ermittlung des effektiven Unternehmensgewinns. Insbesondere sind alle steuerrechtlichen Gestaltungsmöglichkeiten, wie Abschreibungen, Rückstel- 
lungen etc., hinsichtlich ihrer ökonomischen Zweckmäßigkeit zu überprüfen und gegebenenfalls zu korrigieren (Fuest and Kroker, 1989, S. 47). Darüber hinaus gilt es das Problem der korrekten zeitlichen Zuordnung der Steuerleistungen zu lösen. Durch Steuernachzahlungen beziehungsweise -refundierungen wird dies erheblich erschwert.

Die Vorteile dieses Ansatzes liegen in seiner Einfachheit und vor allem in der Verwendung empirischer Unternehmensdaten, die eine vergleichsweise große Realitätsnähe sicherstellen. Zur Beurteilung der bisherigen Entwicklung der tatsächlichen Steuerbelastung ausgewählter Unternehmen erscheint diese Quote gut geeignet. Ex ante-Aussagen, insbesondere über die ökonomischen Anreizwirkungen der Unternehmensbesteuerung, sind jedoch nicht möglich.

\subsubsection{Die modelltheoretische Grenzsteuerbelastung}

Breiten Raum in der Literatur zur Messung der Unternehmenssteuerbelastung nehmen effektive Grenzsteuersätze ein. Im Mittelpunkt steht dabei die Ermittlung der Steuerbelastung der Erträge eines zusätzlichen (hypothetischen) Investitionsprojektes anhand eines neoklassischen Kapitalmarkt-Gleichgewichtsmodells. Ausgehend vom Konzept der Kapitalnutzungskosten in den 60er Jahren (insbesondere Hall and Jorgenson, 1967) erlangte dieser Ansatz seine international größte Beachtung Mitte der 80er Jahre durch die umfangreiche empirische Untersuchung von King and Fullerton (1984). Die von King und Fullerton (weiter-) entwickelte Methodik bildet seitdem die Basis für zahlreiche empirische Untersuchungen. Zu erwähnen sind insbesondere Organisation for Economic Co-operation and Development (1991), Commission of the European Communities (1992) sowie für Österreich jüngst Felderer and Koman (1998) und Sendlhofer and Winner (1998).

Vom Grundkonzept her ist dieser Ansatz einfach. Ausgangspunkt bildet die Überlegung, daß im Kapitalmarktgleichgewicht ein (marginales) Investitionsprojekt ohne Besteuerung gerade so viel Ertrag erwirtschaften muß, wie eine alternative Veranlagung auf dem Kapitalmarkt erbringt. Das heißt, ein Kapitalgeber ist indifferent zwischen der Finanzierung des Investitionsprojektes und einer alternativen Veranlagung. Eine Besteuerung der Erträge des Investitionsprojekts kann als Anstieg der Kapitalnutzungskosten gesehen werden und erhöht somit die (im Gleichgewicht) notwendige interne Rendite des Investitionsprojekts. „... a marginal effective tax is the difference between the internal rates of return on two cash flows associated with an investment. The first is the cash flow before tax rules have been applied, and the second is the cash flow after taxes." (Bradford and Stuart, 1986, S. 4)

In der Terminologie von King and Fullerton (1984), S. 7ff lassen sich die Zusammenhänge folgendermaßen darstellen. Der Steuerkeil (,tax wedge“) $(w)$ einer marginalen Investition ergibt sich als Differenz zwischen der internen Rendite vor Steuern $(p)$ und der Rendite nach Steuern $(s)$, die dem Kapitalgeber zufließt,

$$
w=p-s .
$$

Die interne Rendite vor Steuern $(p)$ stellt die Kapitalnutzungskosten dar. Diese hängen vom realen Kapitalmarktzinssatz $(r)$ ab (zur Kapitalnutzungskostenfunktion im Detail 
vgl. King and Fullerton, 1984, S. 18ff).

$$
p=c(r) \text {. }
$$

Der reale Kapitalmarktzinssatz $(r)$ ergibt sich als Differenz zwischen dem nominalen Marktzinssatz $(i)$ und der Inflationsrate $(\pi)$

$$
r=i-\pi
$$

Die Rendite nach Steuern $(s)$ wiederum hängt von der (marginalen) Steuerbelastung des Kapitalgebers (Grenzsteuerbelastung von Zinseinkünften $m$ und Vermögen $w_{p}$ ) sowie dem realen Kapitalmarktzinssatz $(r)$ und der Inflationsrate $(\pi)$ ab

$$
s=(1-m)(r+\pi)-\pi-w_{p} .
$$

Bezieht man schließlich den Steuerkeil $(w)$ auf die interne Rendite vor Steuern $(p)$ erhält man den effektiven Grenzsteuersatz $(t)$

$$
t=\frac{p-s}{p} .
$$

Wenn keine Unternehmenssteuern erhoben werden $(w=0)$ müssen die interne Rendite vor Steuern beziehungsweise die Kapitalnutzungskosten $(p)$ dem realen Kapitalmarktzinssatz $(r)$ sowie der Rendite nach Steuern $(s)$ entsprechen

$$
p=r=s
$$

Die konkrete Höhe des effektiven Grenzsteuersatzes hängt nun entscheidend von den Modellannahmen ab, insbesondere hinsichtlich des jeweils geltenden Steuersystems, der Art des Investitionsgutes und seiner Finanzierung sowie der Branche und von der Besteuerung des Kapitalgebers. Darüber hinaus bestimmt die (notwendige) Vorgabe eines der drei Parameter $(p, r, s)$ das Ergebnis. Beispielsweise bildet die Vorgabe des realen Kapitalmarktzinssatzes $(r)$ die Steuerbelastung der Einkommen des Kapitalgebers ab und ermöglicht so direkte Aussagen über die Verzerrung der Kapitalallokation durch die Besteuerung (,,fixed-r case“).

Steuerseitig werden regelmäßig alle direkten Steuern auf Ebene des Unternehmens (insbesondere Körperschaftsteuer) und des Kapitalgebers (Steuern auf Zinsen, Dividenden und Kapitalgewinne) sowie die wichtigsten Investitionsförderungs- und Abschreibungsmöglichkeiten einbezogen. Grundsätzlich ließe sich dieser Ansatz auch um weitere das Investitionsprojekt betreffende Abgaben erweitern, wie beispielsweise um Unternehmensbeiträge zur Sozialversicherung oder Umweltabgaben (Gérard et al., 1997).

Die Ermittlung der marginalen Steuerbelastung einzelner Investitionsprojekte kann schließlich in drei Schritten erfolgen (Schneider, 1988, S. 332ff):

1. Berechnung des Unternehmenssteuerkeils, das heißt, der aus dem zugrundegelegten Investitionsprojekt resultierenden Steuerwirkungen im Unternehmen in Bezug auf einen ,entscheidungsneutralen“ Gewinn. Die Steuerbelastung der Investitionsfinanzierung bleibt dabei unberücksichtigt. 
2. Berechnung der nominellen steuerbedingten (zusätzlichen) Finanzierungskosten.

3. Berechnung der gesamten effektiven Grenzsteuerbelastung durch Verbindung von Schritt 1 und 2.

Die (durchschnittliche) effektive Steuerbelastung der volkswirtschaftlichen Einkünfte aus Unternehmertätigkeit (marginale Erhöhung des gesamten volkswirtschaftlichen Kapitalstocks) kann schließlich nur als gewichteter Durchschnitt der nach Art des Investitionsgutes, Branche, Finanzierungsform und Kapitalgeber unterschiedlichen Grenzsteuersätze der einzelnen Investitionsprojekte ermittelt werden.

Der zentrale Vorteil dieses Ansatzes liegt darin, daß er auf einer modelltheoretisch fundierten Abbildung der zugrundeliegenden ökonomischen Zusammenhänge zwischen Besteuerung und Investitionsentscheidung basiert. Durch die Berechnung von Grenzsteuersätzen sind ex ante-Aussagen über die Beeinflussung (allokative Verzerrung) von Unternehmensentscheidungen durch die Besteuerung möglich. Dementsprechend findet diese Methode vor allem in internationalen Vergleichen über die Unternehmensbesteuerung als Standortkriterium Anwendung (King and Fullerton, 1984; Organisation for Economic Co-operation and Development, 1991; Commission of the European Communities, 1992; Felderer and Koman, 1998; Sendlhofer and Winner, 1998).

Aus dem Modellcharakter dieses Ansatzes resultieren jedoch auch wesentliche Nachteile. Die Höhe der Steuerbelastung hängt nämlich entscheidend von den zugrundegelegten Modellannahmen ab. Neben der Voraussetzung von vollkommenem Wettbewerb führen vor allem rigide Annahmen hinsichtlich der einbezogenen Elemente und der zukünftigen Entwicklung des Steuersystems - Verhaltensänderungen durch die Besteuerung sind in der Regel ausgeblendet - zu einer eingeschränkten Allgemeingültigkeit und Praxisrelevanz der Ergebnisse. Darüber hinaus reagieren die Steuerquoten sensibel auf Variationen in den Modellannahmen (Bradford and Stuart, 1986, S. 4). Insbesondere gilt das für die abgeleiteten globalen länderweisen Steuerbelastungsquoten.

Schließlich ist dieser Ansatz infolge der Modelleigenschaften (vor allem ex anteOrientierung und aufwendige Berechnung) auch weniger geeignet für eine Abbildung der (historischen) Entwicklung der Steuerbelastung, wie sie oftmals in finanzpolitischen Studien erfolgt.

\subsection{Makroökonomische Steuerquoten}

Im Gegensatz zu mikroökonomischen (Grenz-) Steuersätzen basieren makroökonomische Steuerquoten auf einem sehr einfachen methodischen Konzept. Ähnlich der gebräuchlichen allgemeinen Steuerquote (gesamtes Steueraufkommen als Anteil am BIP) sind diese Quoten definiert als Verhältnis von gesamtwirtschaftlichem Aufkommen an Unternehmenssteuern (beziehungsweise Steuern auf Kapitaleinkommen) zum volkswirtschaftlichen Aggregat der Unternehmenseinkommen (beziehungsweise Kapitaleinkommen). Sie werden auf der Grundlage von VGR-Daten ermittelt. Dementsprechend basieren sie auf empirischen Werten und sind ex post-orientiert.

Die zentrale Frage bei der Ermittlung von Makroquoten ist die Abgrenzung der einzubeziehenden Steuern und der Bemessungsgrundlage. Dabei ist zu unterscheiden zwischen 
„einfachen“ Makroquoten, die im Wesentlichen die Konventionen der Verteilungsrechnung der VGR akzeptieren, und ,bereinigten“ Makroquoten, die einen Versuch darstellen, die in den VGR ausgewiesenen Größen in Richtung einer dem Leistungsfähigkeitsprinzip entsprechenden Größe für die Gesamtheit der Unternehmenseinkünfte zu korrigieren. Im Falle der einfachen Makroquoten wird oft auch von impliziten Steuersätzen gesprochen. Für die bereinigten Makroquoten gibt es keine allgemein akzeptierte Bereinigungsmethode, sondern nur mehr oder minder tief gehende Bereinigungsversuche.

\section{Möglichkeiten der Messung der Unternehmenssteuer- belastung auf Basis der österreichischen VGR}

Um die Möglichkeiten - aber auch Schwierigkeiten - der Messung der Steuerbelastung von Unternehmen auf VGR-Basis konkret zeigen zu können, gehen die folgenden Ausführungen von den österreichischen Gegebenheiten aus. Bevor jedoch auf die Makroquoten im Einzelnen eingegangen wird, ist es hilfreich sich mit den Konzepten der österreichischen VGR vertraut zu machen, insbesondere mit der Abgrenzung des volkswirtschaftlichen Betriebsüberschusses und der Einkünfte aus Besitz und Unternehmung.

\subsection{Volkswirtschaftlicher Betriebsüberschuß und Einkünfte aus Be- sitz und Unternehmung}

Als gesamtwirtschaftliches Informationssystem stellen die VGR sicherlich das wichtigste empirische Analyseinstrument zur Beantwortung makroökonomischer Fragestellungen dar. Unter Berücksichtigung der Komplexität der Zusammenhänge in einer Volkswirtschaft und dem Anspruch einer konsistenten und überschaubaren Darstellung dieser Zusammenhänge, müssen die VGR (international vergleichbaren) Standards und Konventionen entsprechen (Brümmerhoff, 1995).

Im Bereich wirtschaftlicher Aktivitäten stellen die VGR auf das hierarchische Begriffspaar Betrieb (Ebene I) und Unternehmen (Ebene II) ab (,System-Dualismus“). Dabei entspricht jedem Unternehmen zumindest ein Betrieb. Das bisherige Konzept der österreichischen (amtlichen) VGR ist ausschließlich betriebsbezogen. Direkte Daten auf Unternehmensebene, insbesondere über die Einkommens- und Finanzsphäre, fehlen, da diesbezügliche Erhebungen - abgesehen von der Statistik der Rechnungsabschlüsse der Aktiengesellschaften - bisher nicht durchgeführt wurden. Für die Darstellung von Unternehmensdaten hat das zur Folge, daß diese aus den Betriebsdaten abgeleitet sind. $\mathrm{Zu}$ beachten ist jedoch, daß dem Unternehmenssektor nur Betriebe einer privaten oder öffentlichen Kapitalgesellschaft (AG und GesmbH) oder „Quasikapitalgesellschaft“ (z.B. Genossenschaft oder Sparkasse) zugerechnet werden. Die übrigen Betriebe (von selbständig Erwerbstätigen oder Personengesellschaften) werden als „Unternehmerhaushalte“ unter dem (privaten) Haushaltssektor subsumiert (Franz, 1994).

Erst mit der Umstellung auf das ESVG 1995 (derzeit ESVG 1979) ändert sich diese Situation. Das ESVG 1995 sieht nämlich eine Gliederung in die Sektoren nicht-finanzielle Kapitalgesellschaften, finanzielle Kapitalgesellschaften, Staat, private Haushalte, private Organisationen ohne Erwerbszweck und übrige Welt verpflichtend vor. Dazu wur- 
den erstmals für 1995 auch direkte Erhebungen auf der Ebene von Unternehmen durchgeführt. Erste Daten für Österreich werden jedoch frühestens nach der Jahrtausendwende veröffentlicht werden.

Hinsichtlich der Erfassung der Zahlungsströme in den VGR gilt insbesondere im Bereich der Einkommen aus Besitz und Unternehmung das ,accrual“"-Prinzip - im Gegensatz zum „,cash“-Prinzip, wie beispielsweise in den öffentlichen Finanzstatistiken. Das heißt, daß für den Zeitpunkt einer VGR-Buchung der Rechtsanspruch und nicht die tatsächliche Zahlung maßgeblich ist.

\subsubsection{Volkswirtschaftlicher Betriebsüberschuß}

Entsprechend dem System-Dualismus der VGR ergibt sich der volkswirtschaftliche Betriebsüberschuß aus der Summe der Betriebsüberschüsse aller marktproduzierenden Industrien abzüglich der imputierten Bankgebühr. Konventionsgemäß werden nämlich Zinszahlungen bei den Marktproduzenten nicht erfaßt, die Zinserträge der Banken jedoch schon. Deshalb muß die Summe der Betriebsüberschüsse über alle Industrien global um den Zinsensaldo des Bankensektors bereinigt werden, um Doppelerfassungen zu korrigieren.

Die Betriebsüberschüsse der einzelnen Industrien (Saldo des jeweiligen Produktionskontos) wiederum ergeben sich aus der Summe der Brutto-Wertschöpfungen der einzelnen Wirtschaftsbereiche ergänzt um diverse Imputationen für Eigenleistungen, die nicht über Märkte angeboten werden, beispielsweise für Leistungen im Bereich des Bau- und Wohnungswesens, sowie abzüglich der Löhne und Gehälter (inklusive Arbeitgeberbeiträge zur Sozialversicherung), der volkswirtschaftlichen Abschreibungen und des Saldos aus indirekten Steuern und Subventionen (Kramer, 1988).

\subsubsection{Einkünfte aus Besitz und Unternehmung}

Das Volkseinkommen setzt sich aus den Arbeitseinkommen (Brutto-Entgelte für unselbständige Arbeit) und den Unternehmens- und Besitzeinkommen zusammen (Abbildung 2). Während für die Arbeitseinkommen originäre Daten zur Verfügung stehen, stellt die Gesamtheit der Einkünfte aus Besitz und Unternehmung (EBU) lediglich eine Residualgröße dar.

Wie bereits erwähnt ist der Einkommensbegriff der VGR von der Produktionsseite (Entstehungsrechnung) her bestimmt. Die Summe der EBU wird aus den Betriebsdaten abgeleitet. Gemäß dem den VGR zugrunde liegenden Wirtschaftskreislaufmodell müssen im Aggregat die EBU dem volkswirtschaftlichen Betriebsüberschuß entsprechen. Die einzige Korrekturnotwendigkeit ergibt sich aus dem Faktum, daß der Entstehungsrechnung das Inlandskonzept und der Verteilungsrechnung das Inländerkonzept zugrunde gelegt ist (Haslinger, 1998, S. 46ff). Zur Ermittlung der EBU (ohne Zinsen für die Staats- und Konsumentenschuld) muß daher der volkswirtschaftliche Betriebsüberschuß um den Saldo der Faktoreinkommen an das und aus dem Ausland bereinigt werden.

Für die der Untersuchung zugrunde liegenden Fragestellung stellt die Verteilung der EBU die zentrale Einschränkung dar. Dabei müssen infolge der (noch) fehlenden Sektorengliederung und der Konventionen der österreichischen VGR zum Teil erhebliche 


\begin{tabular}{|c|c|c|c|c|c|c|}
\hline \multirow{3}{*}{$\begin{array}{l}\text { Brutto-Entgelte } \\
\text { für unselbst- } \\
\text { ständige Arbeit }\end{array}$} & \multicolumn{6}{|c|}{ Einkünfte aus Besitz und Unternehmung } \\
\hline & \multirow[b]{2}{*}{$\begin{array}{l}\text { unverteilte } \\
\text { Gewinne } \\
\text { der Kapital- } \\
\text { gesell- } \\
\text { schaften }\end{array}$} & \multirow{2}{*}{$\begin{array}{c}\text { Einkünfte } \\
\text { des Staates } \\
\text { aus Besitz } \\
\text { und Unter- } \\
\text { nehmung } \\
\text { (ohne Zinsen } \\
\text { für die } \\
\text { Staatsschuld) }\end{array}$} & \multicolumn{4}{|c|}{$\begin{array}{l}\text { persönliche EBU (ohne Zinsen } \\
\text { für die Konsumentenschuld) }\end{array}$} \\
\hline & & & $\begin{array}{l}\text { Ein- } \\
\text { künfte } \\
\text { der } \\
\text { Land- } \\
\text { und } \\
\text { Forstw. }\end{array}$ & $\begin{array}{c}\text { Ein- } \\
\text { künfte } \\
\text { der } \\
\text { Gew.- } \\
\text { betriebe }\end{array}$ & $\begin{array}{l}\text { Ein- } \\
\text { künfte } \\
\text { der } \\
\text { freien } \\
\text { Berufe }\end{array}$ & $\begin{array}{l}\text { Besitz- } \\
\text { ein- } \\
\text { künfte }\end{array}$ \\
\hline
\end{tabular}

Abbildung 2: Verteilung des Volkseinkommens

Unschärfen in Kauf genommen werden. Lediglich die Besitz- und Unternehmenseinkünfte des Staates können aus den Rechnungsabschlüssen des öffentlichen Sektors unmittelbar abgeleitet werden. Die Verteilung der übrigen Einkünfte orientiert sich an verschiedenen anderen Quellen innerhalb und außerhalb der VGR. Herangezogen werden beispielsweise die Entstehungsrechnung (Einkünfte der Land- und Forstwirtschaft, Einkünfte aus Vermietung und Verpachtung), die Aktiengesellschaftsstatistik (unverteilte Gewinne der Kapitalgesellschaften), die Einkommensteuerstatistik (Einkünfte der freien Berufe, sonstige Besitzeinkünfte) sowie Daten der Oesterreichischen Nationalbank (Einkünfte aus Kapitalvermögen). Schließlich werden in den Einkünften der Gewerbebetriebe alle ,nicht verteilbaren“ Besitz- und Unternehmenseinkommen zusammengefaßt. Eine befriedigende Zuordnung der Besitzeinkünfte der sogenannten Unternehmerhaushalte zum Haushaltsoder zum Unternehmenssektor ist nicht möglich.

Aufgrund der genannten Einschränkungen der (österreichischen) VGR sind die konkreten Werte einzelner Bereiche, insbesondere der persönlichen EBU mit Vorsicht und für Fragen nach der Steuerbelastung von Unternehmenseinkünften jedenfalls nicht ,unbereinigt" $\mathrm{zu}$ verwenden.

\subsection{Die implizite Steuerbelastung der Kapitaleinkommen}

Aufbauend auf der Überlegung, die gesamtwirtschaftliche Steuerbelastung den einzelnen makroökonomischen Aggregaten beziehungsweise Produktionsfaktoren zuzuordnen, hat sich die Berechnung von so genannten impliziten Steuerquoten etabliert (beispielsweise Eurostat, 1998; Felderer and Koman, 1998). Mit Hilfe dieser Quoten können grobe Aussagen über die makroökonomische Verteilung der Steuerbelastung gemacht werden. Bei der Interpretation dieser Quoten sind jedoch einerseits die Konventionen der VGR zu beachten (siehe Abschnitt 3.1) und andererseits die Tatsache, daß nicht alle Steuern den einzelnen makroökonomischen Aggregaten eindeutig zugeordnet werden können (Felderer and Koman, 1998, S. 7ff). Deshalb unterscheidet beispielsweise das Eurostat lediglich 
zwischen der Steuerbelastung von Konsum, Arbeit und „sonstigen Produktionsfaktoren“ (Eurostat, 1998, S. 10ff). Präzise Aussagen über die Steuerbelastung der Einkünfte ausschließlich unternehmerischer Aktivitäten sind mit diesen ,einfachen“ impliziten Steuerquoten nicht möglich.

Eine theoretische Fundierung für die impliziten Steuerquoten bieten Mendoza et al. (1994). Entsprechend der Inzidenz im Rahmen eines makroökonomischen Gleichgewichtsmodells wird die (durchschnittliche) effektive Steuerbelastung der drei „Güter“ Konsum, Arbeit und Kapital abgeleitet. Berechnet werden ,ad valorem tax rates that represent the wedges distorting optimal plans in a macroeconomic, representative agent setting by comparing measures of aggregate post- and pre-tax incomes and prices" (Mendoza et al., 1994, S. 320). Die implizite Steuerquote der Kapitaleinkommen $\left(\tau_{k}\right)$ lautet demnach

$$
\tau_{k}=\frac{-q_{k} y_{k}-\left(-p_{k} y_{k}\right)}{-q_{k} y_{k}} .
$$

Dabei ist im Zähler die Differenz des mit Preisen vor Steuern $\left(q_{k}\right)$ und nach Steuern $\left(p_{k}\right)$ bewerteten volkswirtschaftlichen Kapitaleinsatzes $\left(y_{k}\right)$ erfaßt. Bezogen wird dieser makroökonomische Steuerkeil (im Unterschied zum mikroökonomischen Steuerkeil, siehe Abschnitt 2.2.2) auf die Gesamtheit aller Kapitaleinkünfte zu Vor-Steuerpreisen $\left(q_{k} y_{k}\right)$. Dabei resultiert das negative Vorzeichen des volkswirtschaftlichen Kapitaleinsatzes $\left(y_{k}\right)$ aus seiner Eigenschaft als Inputfaktor (Mendoza et al., 1994, S. 301f).

Zur konkreten Berechnung der Steuerquote gehen Mendoza et al. (1994) davon aus, daß der Zähler durch die Summe der jährlichen Kapitalsteueraufkommen und der Nenner durch die Summe der entsprechenden Steuerbemessungsgrundlagen angenähert werden können. Auf Basis der österreichischen VGR berechnet sich die implizite Steuerquote der Kapitaleinkommen $\left(\tau_{k}\right)$ als

$$
\tau_{k}=\left(\frac{\sum \text { Steuern auf EBU }}{\text { EBU }}\right) 100
$$

Als aufkommensstärkste Steuern sind im Zähler auf Bundesebene die Körperschaftsteuer, die veranlagte Einkommensteuer, die Kapitalertragsteuern I und II und die Grunderwerbsteuer sowie auf Ebene der nachgeordneten Gebietskörperschaften die Grundsteuern A und B und die Interessentenbeiträge von Grundstückseigentümern und Anrainern zu erfassen.

Die Vorteile der einfachen impliziten Steuerquoten liegen vor allem

- im einfachen methodischen Konzept,

- in der Verwendung standardisierter empirischer Daten und ihre vergleichsweise leichte Verfügbarkeit (auch als Zeitreihen),

- in der Geschlossenheit des Systems impliziter Steuerquoten und

- in der impliziten Abbildung aller steuerrechtlichen Regelungen.

Diesen Vorteilen stehen jedoch gewichtige Nachteile gegenüber. Aus dem Fehlen eines modellierten - mikroökonomisch fundierten - Wirkungszusammenhanges zwischen den (makroökonomischen) Kapitaleinkünften und den Steuern resultiert, daß Aussagen über 
die ökonomischen Anreizwirkungen der Kapitalbesteuerung nicht möglich sind. Generell stellt sich die Frage, ob zwischen den Makrogrößen der VGR und den zugrunde liegenden Mikrogrößen überhaupt eindeutige Beziehungen bestehen (Brümmerhoff, 1995, S. 215).

Weiters erlauben implizite Steuerquoten aufgrund der Heterogenität der (zum Teil untrennbar) gemeinsam erfaßten Kapitaleinkünfte (siehe Abschnitt 3.1.2) keine Aussagen über die Verteilung der Steuerlast auf einzelne Kapitaleinkunftsarten. Aufspaltungen sind allenfalls in relativ grobe Kategorien sinnvoll, wie beispielsweise Kapitaleinkünfte im engeren Sinn und Einkommen aus selbständiger Arbeit (Felderer and Koman, 1998, S. 7ff). Damit wird dem Umstand entsprochen, daß die persönlichen EBU natürlich nicht nur eine Abgeltung von Kapitaleinsatz, sondern auch von „Unternehmerarbeit" darstellen. Die Interpretation der impliziten Steuerbelastung der Einkommen aus selbständiger Arbeit ist jedoch nicht unproblematisch, einerseits aufgrund der erwähnten „Residualfunktion“ der Einkünfte aus Gewerbebetrieb in den VGR und andererseits aufgrund der steuerrechtlichen Einkommenspauschalierungen in der Land- und Forstwirtschaft.

\subsection{Die implizite Steuerbelastung von Unternehmenseinkünften}

Als Indikator für die Steuerbelastung der Einkünfte aus Unternehmertätigkeit weist die implizite Steuerbelastung der Kapitaleinkommen entscheidende Nachteile auf. Diese resultieren vor allem aus dem gewählten methodischen Ansatz: der Aufspaltung der gesamtwirtschaftlichen Steuerbelastung auf die Einkommenskategorien nach Produktionsfaktoren. Gemäß dem finanzwissenschaftlichen Leistungsfähigkeitsprinzip müßte jedoch ein geeigneter Indikator an den Jahreseinkünften (ausgeschüttete und zurückbehaltene Gewinne) aller Unternehmen einer Volkswirtschaft ansetzen, die im Steuerrecht eine Entsprechung finden. Erste Ansätze dafür bieten Franz and Teufelsbauer (1979), Littmann (1987) und Fuest and Kroker (1989).

\subsubsection{Ermittlung der volkswirtschaftlichen Unternehmenseinkünfte}

Auf Basis der VGR lassen sich für die Ermittlung der volkswirtschaftlichen Unternehmenseinkünfte zwei (eng miteinander zusammenhängende) Ausgangspunkte heranziehen: einerseits der volkswirtschaftliche Betriebsüberschuß als makroökonomische Gewinngröße der Unternehmensaktivitäten (siehe Abschnitt 3.1.1) und andererseits die Einkünfte aus Besitz und Unternehmung als Residuum zu den Einkommen aus unselbständiger Arbeit (siehe Abschnitt 3.1.2). Die Konventionen der VGR und die Abgrenzung des Einkommensbegriffs im (österreichischen) Steuerrecht erfordern jedoch in beiden Fällen erhebliche Bereinigungen.

Geht man vom volkswirtschaftlichen Betriebsüberschuß aus, sind jedenfalls folgende Korrekturen vorzusehen:

- Erstens sind jene wirtschaftlichen Aktivitäten (Betriebe) auszuscheiden, die nicht besteuert werden und daher für die Berechnung der impliziten Steuerbelastung nicht relevant sind.

- Zweitens sind jene Einkommenskategorien hinzuzurechnen, die als Unternehmenseinkünfte zu werten sind, im volkswirtschaftlichen Betriebsüberschuß aber nicht erfaßt sind. 
- Drittens sind Anpassungen infolge der unterschiedlichen Behandlung von Unternehmenseinkünften (-gewinnen) in den VGR und im Steuerrecht notwendig.

Geht man hingegen von der sektoralen Verteilung der EBU aus (siehe Abbildung 2), sind vorerst alle Einkommensströme auszuscheiden, die entweder keine Unternehmenseinkünfte darstellen oder in Österreich nicht besteuert werden. Das sind

- die Besitzeinkünfte des Staatssektors (insbesondere die Einkünfte aus Zinsen und Währungstauschverträgen) - die öffentlichen Unternehmenseinkünfte jedoch nur insoweit als sie von der Steuer ausgenommen sind - und

- die Besitzeinkünfte des Haushaltssektors, soweit sie nicht aus Unternehmensbeteiligungen stammen.

Die Schwierigkeit bei diesem Ansatz liegt in der Ermittlung der auszuscheidenden Besitzeinkünfte des Haushaltssektors. Insbesondere ist im Rahmen der VGR die erforderliche Aufspaltung der Besitzeinkünfte der so genannten Unternehmerhaushalte in Einkommen aus Unternehmensaktivitäten und sonstige „Haushalts“-Einkommen nicht möglich (siehe Abschnitt 3.1.2).

Darüber hinaus sind analoge Korrekturen notwendig, wie beim volkswirtschaftlichen Betriebsüberschuß unter erstens und drittens erwähnt. Zusätzlich erfordert das in der Verteilungsrechnung geltende Inländerkonzept, daß im Ausland erzielte und versteuerte Einkommen inländischer Unternehmen abzuziehen und daß im Inland erzielte Einkommen ausländischer Unternehmen hinzuzuzählen sind.

In Anbetracht der genannten Probleme bei einer sektorgerechten Aufspaltung der Besitzeinkünfte ist dem Ansatz - ausgehend vom volkswirtschaftlichen Betriebsüberschuß - der Vorzug zu geben. Entsprechend obiger Grobstruktur sind im Einzelnen folgende Korrekturschritte vorzunehmen:

1. Bereinigung um im volkswirtschaftlichen Betriebsüberschuß erfaßte, aber nicht besteuerte Einkünfte bestimmter wirtschaftlicher Aktivitäten:

- Imputationen (für den Wohnbau, den Mietwert für Eigenheime und die Privatzimmervermietung)

- Gewinndifferenzen zwischen VGR und Steuerstatistik bei pauschalierten Betrieben der Land- und Forstwirtschaft

In den VGR werden die Gewinne der gemäß Steuerrecht pauschalierten Betriebe der Land- und Forstwirtschaft voll erfaßt. Die Differenz zwischen in den VGR ausgewiesenen Gewinnen und der Summe der Pauschalbeträge gemäß Steuerstatistik ist in Abzug zu bringen.

- Gewinne steuerbefreiter Unternehmen

Bestimmte Unternehmen, die in den VGR voll erfaßt sind, unterliegen keiner Steuerpflicht, wie die Österreichischen Bundesbahnen und bis 1991 die Oesterreichische Nationalbank sowie - solange sie direkt im öffentlichen Haushalt integriert sind - die staatlichen Monopolbetriebe. Daneben gibt es noch weitere Unternehmen, die von der Körperschaftsteuer befreit sind (Beteiligungsfondsgesellschaften, diverse Genossenschaften etc.). Allfällige Gewinne von Betrieben dieser Unternehmen sind in Abzug zu bringen. 
- Einkommen in der Schattenwirtschaft

Im Rahmen der Entstehungsrechnung der VGR werden aus Vollständigkeitsgründen Korrekturen und Zuschätzungen vorgenommen. Diese setzen sich aus Vollständigkeitskorrekturen infolge Untererfassung und unterschiedlicher Vorleistungsabgrenzungen in den VGR und in den Unternehmensbuchhaltungen sowie aus Zuschätzungen der Schattenwirtschaft zusammen. Letztere teilen sich in die „Ohne Rechnungs“-Geschäfte der Unternehmen und in die "Schwarzarbeit“ der Unselbständigen. Beide Einkommenskategorien werden jedoch den Unternehmenseinkünften zugezählt. Insgesamt sind daher die Zuschätzungen für die Schattenwirtschaft und die Korrekturen infolge der definitorischen Unterschiede wieder in Abzug zu bringen. Lediglich die Zuschätzungen aufgrund der unvollständigen Erfassung der Unternehmen sind davon nicht betroffen (zu den Vollständigkeitskorrekturen im Detail Schwarzl and Kaßberger, 2000).

2. Ergänzung um Unternehmenseinkünfte, die im volkswirtschaftlichen Betriebsüberschuß nicht erfaßt sind:

- Zinszahlungen der privaten und öffentlichen Haushalte an marktproduzierende Betriebe (netto, d.h. bereinigt um die Zinserträge der Haushalte)

- Saldo der Faktoreinkommen (Zinsen und sonstige Faktoreinkommen) mit dem Ausland; ausgenommen jene für die gemäß Doppelbesteuerungsabkommen bereits im Ausland Steuern geleistet wurden

3. Anpassungen infolge der unterschiedlichen Behandlung von Unternehmenseinkünften (-gewinnen) in den VGR und im Steuerrecht

- Berücksichtigung der unterschiedlichen Behandlung von Abschreibungen Die VGR gehen im Vergleich zum Steuerrecht von durchwegs deutlich längeren Nutzungszeiträumen für Anlagegüter aus. Darüber hinaus gibt es auch Unterschiede in den verwendeten Bewertungsprinzipien. Während im Steuerrecht in der Regel das Anschaffungs- beziehungsweise Herstellungskostenprinzip gilt, gehen die VGR stets von Wiederbeschaffungspreisen aus. Die jährlichen volkswirtschaftlichen Abschreibungen sind daher stets geringer als die Summe der steuerrechtlichen Abschreibungen. Wieweit dieser positive Saldo jedoch zu bereinigen ist, läßt sich schwer beurteilen, da die Frage nach der ökonomisch richtigen Höhe der Abschreibungen nicht beantwortet werden kann (Fuest and Kroker, 1989, S. 21).

- Berücksichtigung von in den VGR nicht vorgesehenen gewinnmindernden Positionen des Steuerrechts

Im Unterschied zu den VGR sieht das (österreichische) Steuerrecht verschiedene Positionen vor, die den zu versteuernden Unternehmensgewinn mindern. Dazu zählen

- Rückstellungen, Rücklagen und die Bildung stiller Reserven (insbesondere Abfertigungs- und Pensionsrückstellung); 
- Wertberichtigungen (beispielsweise infolge von Forderungsausfällen, Lagerabwertungen oder außerordentlichen Abschreibungen);

- Investitionsbegünstigungen (Investitionsfreibetrag und bis 1993 die Investitionsrücklage) und

- Beiträge zu Berufskammern, zur Sozialversicherung und zu sonstigen Pflichtversicherungen der Selbständigen.

Geht man davon aus, daß diese gewinnmindernden Positionen ökonomisch gerechtfertigt sind, dann weisen die VGR die Unternehmenseinkünfte zu hoch aus. Dementsprechende Abzüge sind vorzusehen. Gleiches gilt bis 1993 für die Gewerbesteuerzahlungen der Betriebe. Diese galten als Betriebsausgabe und waren daher gewinnmindernd.

Ohne zusätzliche statistische Quellen lassen sich einige der genannten Korrekturen des volkswirtschaftlichen Betriebsüberschusses nicht durchführen. Insbesondere bieten die VGR keine Daten über die sektoralen Faktoreinkommensströme. Weiters müssen für die pauschalierten Einkommen der Land- und Forstwirtschaft, die Gewinne steuerbefreiter Betriebe und sämtliche steuerrechtlichen Abzugspositionen - soweit überhaupt verfügbar - alternative Quellen, wie Daten der Oesterreichischen Nationalbank, die amtlichen Steuerstatistiken und die Rechnungsabschlüsse des öffentlichen Sektors herangezogen werden. Der große Nachteil dabei ist jedoch, daß diese Quellen gemeinsam mit den VGR kein integriertes - international vergleichbares - statistisches Gesamtsystem darstellen.

\subsubsection{Die Steuern auf Unternehmenseinkünfte}

Im Vergleich zur Ermittlung der bereinigten volkswirtschaftlichen Unternehmenseinkünfte erscheint die Abgrenzung der Unternehmenssteuern unproblematisch. Folgende Steuern des österreichischen Steuersystems sind einzubeziehen:

- Körperschaftsteuer

- Kapitalertragsteuer

- Gewerbe- und Bundesgewerbesteuer (bis einschließlich 1993)

- veranlagte Einkommensteuer (teilweise)

- Kapitalertragsteuer auf Zinsen (teilweise)

Während die Körperschaftsteuer, die Kapitalertragsteuer (auf Erträge aus Unternehmensbeteiligungen) und die Gewerbesteuern eindeutig den Unternehmenseinkünften zuzurechnen sind, ist das bei der veranlagten Einkommensteuer und der Kapitalertragsteuer auf Zinsen nur eingeschränkt der Fall. Bei den Gewerbesteuern ist jedoch zu beachten, daß die Gewerbesteuerzahlungen bei der Gewinnermittlung abzugsfähig waren.

Der überwiegende Anteil der veranlagten Einkommensteuer dürfte von Unternehmenseinkünften stammen, da ein Großteil der Besitzeinkünfte des Haushaltssektors bereits von der Kapitalertragsteuer auf Zinsen erfaßt wird. Problematisch ist lediglich der infolge der ,synthetischen“ Einhebung schwierig ermittelbare Anteil der Einkommensteuer 
auf „nicht-Unternehmenseinkünfte“ der Unternehmerhaushalte (siehe Abschnitt 3.3.1). Gewisse Hinweise über den Umfang lassen sich jedoch aus der Einkommensteuerstatistik nach schwerpunktmäßigen Einkunftsarten gewinnen (Österreichisches Statistisches Zentralamt, 1998).

Bei der Kapitalertragsteuer auf Zinsen ist davon auszugehen, daß der überwiegende Anteil dem Haushaltssektor zuzurechnen ist. Eine diesbezügliche Korrektur wäre daher vorzusehen. Verläßliche Aussagen darüber erscheinen jedoch aufgrund fehlender Daten ebenfalls nicht möglich.

In Österreich stehen grundsätzlich zwei Datenquellen für die Unternehmenssteuern zur Verfügung: einerseits die amtliche Steuerstatistik (Einkommensteuer-, Körperschaftsteuer- und Gewerbesteuerstatistik; nicht jedoch für die Kapitalertragsteuern) und andererseits die in den VGR ausgewiesenen Steueraufkommen.

Beide Datenquellen weisen Vor- und Nachteile auf. Aus methodischer Sicht spricht vor allem für die Verwendung der Steuerstatistiken, daß dort die Steuern in einem höheren Detaillierungsgrad erfaßt und periodengerecht abgegrenzt sind. Grundlage dafür sind nämlich die Steuerbescheide für das jeweilige Veranlagungsjahr. Dem stehen jedoch zwei gewichtige Nachteile gegenüber: erstens liegen die Daten erst mit beträchtlicher Zeitverzögerung vor (etwa vier Jahre) und zweitens ist ihre Erfassung nicht vollständig. Beispielsweise werden nachträgliche Änderungen der Steuervorschreibungen infolge von Betriebsprüfungen oder noch nicht erledigte Steuerfälle nicht erfaßt und auch nicht nachgetragen.

Die Steuerdaten der VGR sind im Vergleich zur Steuerstatistik wesentlich früher verfügbar. Sie basieren auf den im Bundeshaushalt ausgewiesenen Steuereinnahmen. Gemäß den Konventionen der VGR werden jedoch Korrekturen vorgenommen. Bei der veranlagten Einkommensteuer werden beispielsweise Erstattungsprämien, wie Bausparprämien und Kinderabsetzbeträge, hinzugerechnet (und gleichzeitig als Transfers an die Haushalte gegengebucht). Bei der Körperschaftsteuer werden (ab 1997) Steuerzahlungen für bestimmte Gewinne der Oesterreichischen Nationalbank abgezogen. Darüber hinaus wird aufgrund der Anwendung des ,,accrual“"-Prinzips in den VGR zusätzlich ein „Saldo der fälligen Forderungen“ gemeinsam für alle direkten Bundessteuern ausgewiesen. Zur Erfassung der tatsächlichen Unternehmenssteuerzahlungen sind diese Korrekturen wieder zurückzuführen.

Der zentrale Nachteil insbesondere der Einkommensteuerdaten der VGR ist, daß diese - da sie auf den Kassaeinnahmen des Bundes basieren - eine Mischung aus den Steuereinnahmen vorhergegangener Veranlagungsjahre und aus den Vorauszahlungen für das laufende Jahr darstellen.

\subsubsection{Die bereinigte implizite Steuerquote}

Vor allem aus Aktualitätsgründen ist es - trotz der aufgezeigten Probleme - nahe liegend, zur Ermittlung der impliziten (makroökonomischen) Steuerbelastung der Unternehmenseinkünfte $\left(\tau_{u}\right)$ die Unternehmenssteuern der VGR (ohne Saldo fälliger Forderungen) auf die bereinigten volkswirtschaftlichen Unternehmenseinkünfte zu beziehen. Ohne Berück- 
sichtigung der 1993 ausgelaufenen Gewerbesteuern ergibt sich die Steuerbelastung als

$$
\tau_{u}=\left(\frac{E S t_{u}+K S t_{b}+K E S t I+K E S t I I_{u}}{E U_{b}}\right) 100 .
$$

Im Zähler handelt es sich um die in den VGR ausgewiesene veranlagte Einkommensteuer ( $E S t_{u}$, bereinigt um die Erstattungsprämien und den ESt-Anteil auf Haushaltseinkommen), Körperschaftsteuer ( $K S t_{b}$, ergänzt um die Steuerzahlungen der Oesterreichischen Nationalbank), Kapitalertragsteuer (KESt I auf Erträge aus Unternehmensbeteiligungen) und um den den Unternehmen zurechenbaren Anteil der Kapitalertragsteuer auf Zinsen $\left(K E S t I I_{u}\right)$. Im Nenner stehen die in Abschnitt 3.3.1 abgeleiteten volkswirtschaftlichen Einkünfte aus Unternehmertätigkeit $\left(E U_{b}\right)$.

Neben den dargestellten Problemen bei der Herleitung von Zähler und Nenner ergeben sich zusätzliche Schwierigkeiten bei der Interpretation der Steuerquote selbst.

Aufgrund der Tatsache, daß die veranlagte Einkommensteuer in Österreich einen progressiven Tarifverlauf aufweist, hängt die (durchschnittliche) Gesamtsteuerbelastung naturgemäß auch von der Verteilung der Unternehmenseinkünfte ab. Eine steigende Steuerbelastung muß nicht unbedingt auf Steuererhöhungen oder insgesamt steigende Unternehmenseinkünfte hinweisen, sie kann auch das Resultat einer auseinander driftenden Einkommensverteilung sein.

Weiters bestehen gewisse Einschränkungen der Aussagekraft der dargestellten Steuerquote im Konjunkturverlauf. Erstens bewirkt die im Steuerrecht vorgesehene Möglichkeit eines Verlustausgleichs eine Nivellierung der Steuerleistungen von Unternehmen im Zeitablauf. Zweitens wirkt die angesprochene nicht-periodengerechte Abgrenzung der Unternehmenssteuern in den VGR ebenfalls verzerrend. Mittel- bis langfristig betrachtet sollte jedoch - unter Beachtung der angeführten Einschränkungen - ein zutreffendes Bild der Höhe und Entwicklung der Steuerbelastung möglich sein. Allerdings wird dieser Befund durch häufige Änderungen im österreichischen Steuerrecht (Veranlagungsumstellung bei der Einkommensteuer, Umstellung der Kapitalertragsbesteuerung, Auflassen der Gewerbesteuern etc.) beeinträchtigt. Ohne Rückgriff auf die detaillierten und periodengerechten Daten der Steuerstatistik dürfte daher eine seriöse Interpretation der Entwicklung der bereinigten impliziten Steuerquote nicht möglich sein.

\section{Resümee}

Die vorliegende Arbeit zeigt, daß es zwei grundsätzlich unterschiedliche Methoden zur Messung der effektiven Steuerbelastung von Unternehmen (-seinkünften) gibt. Auf der einen Seite steht der modelltheoretische Zugang zur Berechnung der Grenzsteuerbelastung einzelner Investitionsprojekte beziehungsweise idealtypischer Unternehmen, auf der anderen Seite steht die aus den VGR ermittelte implizite Steuerbelastung der Kapitalbeziehungsweise Unternehmenseinkommen. Die Ergebnisse beider Ansätze sind jedoch nicht vergleichbar, sie liefern Antworten auf unterschiedliche Fragestellungen.

Während bei den mikroökonomischen Steuerquoten Effizienz- und Anreizgesichtspunkte der Besteuerung im Vordergrund stehen, versuchen die makroökonomischen Steuerquoten die tatsächliche (durchschnittliche) Steuerbelastung einzelner volkswirtschaftlicher Aggregate abzubilden. Dementsprechend eignen sich Mikroquoten vor allem zur 
Beurteilung der Auswirkungen der Besteuerung auf Unternehmensentscheidungen, wie sie etwa im Zusammenhang mit internationalen Standortfragen (Investitionsentscheidungen) auftreten. Makroquoten hingegen setzen an der Verteilung der Steuerlast an, sie stellen eine „Verfeinerung“ der globalen Steuerquote dar. Verteilungspolitische Fragen stehen daher im Mittelpunkt dieses Ansatzes. Eine Bewertung der makroökonomischen Auswirkungen steuerpolitischer Maßnahmen (und deren Änderungen in der Vergangenheit) ist damit möglich.

Die Beschäftigung mit der Verteilungsrechnung im System österreichischer VGR zeigt jedoch, daß insbesondere die Abgrenzung der ,persönlichen Einkünfte aus Besitz und Unternehmung“" erhebliche Unschärfen aufweist. Diese spiegeln sich auch in der - daher schwierig zu interpretierenden - impliziten Steuerquote der Kapitaleinkommen wider. Als Indikator für die Steuerbelastung der Einkünfte aus Unternehmertätigkeit ist diese Quote daher kaum geeignet. Ihr unmittelbarer Vergleich mit der Steuerbelastung der Arbeitseinkommen ist problematisch.

Die Diskussion der grundlegenden Ermittlungsmethoden auf Basis der VGR und die vorgeschlagenen Bereinigungsmaßnahmen, vorwiegend bei der Ermittlung der volkswirtschaftlichen Unternehmenseinkünfte, bieten jedoch eine Möglichkeit diese Einschränkungen zu verringern. Die bereinigte implizite Steuerquote kann daher als ein Ansatz gesehen werden, den tatsächlichen Beitrag der Unternehmen zur Staatsfinanzierung verläßlicher zu ermitteln. Bei der derzeitigen Datenlage der offiziellen österreichischen Statistik ist die Berechnung dieser Quote jedoch mit erheblichem Aufwand verbunden und große Datendefizite müssen in Kauf genommen werden. Inwieweit die Umstellung des österreichischen Systems Volkswirtschaftlicher Gesamtrechnungen auf das ESVG 1995 entscheidende Verbesserungen in der Datenverfügbarkeit bringen wird, bleibt erst abzuwarten.

\section{Danksagung}

Ich danke dem anonymen Referee für seine konstruktiven Hinweise. Sie waren für die Überarbeitung des Manuskripts sehr hilfreich. Weiters gilt mein Dank den Herren Mag. Reinhold Schwarzl und DI Walter Stübler im Österreichischen Statistischen Zentralamt sowie Herrn Dr. Anton Rainer im Bundesministerium für Finanzen für ihre wertvolle Unterstützung in der Entstehungsphase der Untersuchung.

\section{Literatur}

D. Bradford and Ch. Stuart. Issues in the Measurement and Interpretation of Effective Tax Rates. Working paper 1975, National Bureau of Economic Research, Cambridge, MA, 1986.

D. Brümmerhoff. Volkswirtschaftliche Gesamtrechnungen. München, Wien, 5th edition, 1995. 
D.S. Callihan. Corporate Effective Tax Rates: a Synthesis of the Literature. Journal of Accounting Literature, 13:1-43, 1994.

Commission of the European Communities. Report of the Committee of Independent Experts on Company Taxation. Brussels, Luxembourg, 1992.

Eurostat. Structures of the Taxation Systems in the European Union, 1970 - 1996. Brussels, Luxembourg, 1998.

B. Felderer and R. Koman. Perspektiven des Wirtschaftsstandortes Österreich aus der Sicht der Steuerpolitik. Studie, Institut für Höhere Studien, Wien, 1998.

A. Franz. Volkswirtschaftliche Gesamtrechnungen - Das statistische System der Makroökonomie. Österreichische Studien zur amtlichen Statistik 1, Österreichisches Statistisches Zentralamt, Wien, 1994.

A. Franz and W. Teufelsbauer. Gewinne im volkswirtschaftlichen und im steuerrechtlichen Rechnungswesen. Wirtschaftspolitische Blätter, (6):16-40, 1979.

W. Fuest and R. Kroker. Unternehmenssteuerlast: 20 oder 70 Prozent? Köln, 1989.

M. Gérard, L. Beauchot, S. Jamaels, and Chr. Valenduc. MESC (Marginal Effective Statutory Charge). An Extension of King-Fullerton Methodology. Diskussionsbeitrag, Serie II 353, Universität Konstanz, Sonderforschungsbereich 178, ,Internationalisierung der Wirtschaft“, Konstanz, 1997.

R.E. Hall and D.W. Jorgenson. Tax Policy and Investment Behavior. American Economic Review, 57:391-414, 1967.

F. Haslinger. Volkswirtschaftliche Gesamtrechnung. München, Wien, 7th edition, 1998.

M.A. King and D. Fullerton. The Taxation of Income from Capital. A Comparative Study of the United States, the United Kingdom, Sweden, and West Germany. Chicago, London, 1984.

I. Kramer. Diskrepanzen zwischen verschiedenen Gewinnkonzepten in Österreich. In Österreichisches Statistisches Zentralamt, editor, Österreichs Volkseinkommen 1987, pages 391-414. Wien, 1988.

K. Littmann. Die Steuerlast der Unternehmen: Positives und Kritisches zu einem Orientierungspunkt der Steuerpolitik. Schriftenreihe der Klaus-Dieter-Arndt-Stiftung e.V. II, Vortrag vor der Klaus-Dieter-Arndt-Stiftung am 17. September 1987 im Wissenschaftszentrum in Bonn, Bonn, 1987.

E.G. Mendoza, A. Razin, and L.L. Tesar. Effective tax rates in macroeconomics. Crosscountry estimates of tax rates on factor incomes and consumption. Journal of Monetary Economics, 34:297-323, 1994.

Organisation for Economic Co-operation and Development. Taxing Profits in a Global Economy. Domestic and International Issues. Paris, 1991. 
Österreichisches Statistisches Zentralamt. Einkommensteuerstatistik 1995. Wien, 1998.

B. Schaden. Effektive Kapitalsteuerbelastung in Europa. Heidelberg, 1995.

D. Schneider. Hochsteuerland Bundesrepublik Deutschland: Die Spannweite effektiver Grenzsteuerbelastungen für Investitionen. Die Wirtschaftsprüfung, 41(11):328-338, 1988.

R. Schwarzl and F. Kaßberger. Zur Vollständigkeit der offiziellen BIP/BSP-Berechnungen. Statistische Nachrichten, (2):142-148, 2000.

R. Sendlhofer and H. Winner. Unternehmensbesteuerung als Standortfaktor - Ein internationaler Vergleich zur Position Österreichs in den neunziger Jahren. Projektbericht, Universität Innsbruck, Institut für Finanzwissenschaft, Innsbruck, 1998.

Adresse des Autors:

Dr. Thomas Kostal

Institut für Finanzwissenschaft

Wirtschaftsuniversität Wien

Augasse 2-6

A-1090 Wien

Tel. +43 $131336-4576$

Fax +43 1 31336-9203

E-mail: thomas.kostal@wu-wien.ac.at 